\title{
Taste Masked Quinine Sulphate Loaded Solid Lipid Nanoparticles for Flexible Pediatric Dosing
}

\author{
Panchaxari Mallappa Dandagia, Seeprarani Prabirkumar Rath ${ }^{{ }^{*}}$, Anand Panchakshari Gadada ${ }^{a}$, Vinayak \\ Shivamurthi Mastiholimath ${ }^{\mathrm{b}}$
}

aDepartment of Pharmaceutics, KLEU's College of Pharmacy, Nehru Nagar, Belgaum - 590 010, India

'Department of Quality Assurance, KLEU's College of Pharmacy, Nehru Nagar, Belgaum - 590 010, India

\begin{abstract}
Introduction: Quinine Sulphate is an antimalarial agent usually indicated in the treatment of chloroquine resistant malaria. Objective: The objective of the present investigation was to prepare quinine sulphate loaded solid lipid nanoparticles by ultrasonic solvent emulsification technique using different surfactants (Tween 80 , Poloxamer 407, Poloxamer 188) in order to mask the bitter taste, thereby improving patient compliance and to provide dose precision and a flexible system that allows dose adaptation according to the body weight. Method: Glyceryl monostearate was used as a lipid (drug to lipid ratio 1:3). The prepared solid lipid nanoparticles were characterized for various parameters like particle size and shape, zeta potential, entrapment efficiency, In vitro evaluation of taste masking efficiency, In vitro drug release, In vitro drug release kinetics. Results and discussion: The mean hydrodynamic diameter of the particle decreased whereas the entrapment efficiency increased with an increase in the surfactant concentration. Higher surfactant concentration showed faster In vitro release. The formulations showed negligible release at $\mathrm{pH} 6.8$ and almost $100 \%$ release at $\mathrm{pH} \mathrm{1.2,} \mathrm{which} \mathrm{is} \mathrm{desirable} \mathrm{so} \mathrm{as} \mathrm{to} \mathrm{mask} \mathrm{the}$ taste by delaying the release during administration without hampering the drug release in stomach. Formulation F9 containing $2 \% \mathrm{w} / \mathrm{v}$ poloxamer 188 was selected as the optimized formulation as it showed high entrapment efficiency and negligible release in Simulated Salivary Fluid (SSF) pH 6.8 when compared to pure drug but showed almost $100 \%$ release at $\mathrm{pH}$ 1.2. Conclusion: It can be concluded that quinine sulphate was proven to be a suitable candidate for formulating solid lipid nanoparticles to achieve better patient compliance among pediatric and geriatric populations by masking the bitter taste and avoiding the difficulty in swallowing.
\end{abstract}

Key words: Quinine Sulphate, Taste masking, Solid Lipid Nanoparticles, Glyceryl monostearate, Poloxamer 188, Poloxamer 407.

\section{INTRODUCTION}

Solid Lipid Nanoparticles (SLNs) have been reported as an alternative drug delivery system to traditional polymeric nanoparticles. A clear advantage of SLNs over polymeric nanoparticles is that the lipid matrix is made from physiologically tolerated lipid components, which decreases the potential for acute and chronic toxicity. At room temperature the particles are in the solid state. SLNs combine the advantages of polymeric nanoparticles, fat emulsions and liposomes. They can be produced on a large industrial scale by high-pressure homogenization, with low toxicity potential like emulsions and liposomes, produce sustained release due to their solid matrix, similar to polymeric nanoparticles and can effectively target specific tissues after parenteral administration. SLNs are easily prepared nanoparticles made from inexpensive, safe, stable and biodegradable materials and can be loaded internally or externally with active pharmaceutical ingredients for controlled delivery. ${ }^{1}$

Quinine, an antimalarial agent, is usually given orally as sulphate or hydrochloride salt, or in case of severe malaria when the patient is unable to take oral medication, it is administered parenterally by slow intravenous infusion as dihydrochloride salt. ${ }^{2}$
Submission Date : 06-05-14 Revision Date : :17-09-14 Accepted Date : :26-11-14

DOI: $10.5530 /$ ijper.48.4s.12 Correspondence Address Miss Seeprarani Rath Department of Pharmaceutics, KLEU's College of Pharmacy,

Belgaum- 590010, Karnataka, India.E-mail: seepra@ rocketmail.com

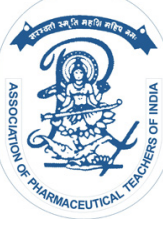

www.ijper.org 
Quinine is rapidly absorbed when taken orally and peak plasma concentrations are reached within 1-3 h. For oral administration, quinine is commercially available as tablets in most of cases containing sulfate or hydrochloride salts in a dose of 200 or $300 \mathrm{mg}$ per tablet. Although this molecule has been commercially used for decades, very few liquid formulations are available. Tablet breaking, practiced to adapt the dose to the body weight of the children, may not provide an accurate dose. ${ }^{3}$

Intramuscular injection may cause lower limb paralysis if administered mistakenly into the sciatic nerve. Thus, the rectal use was used to overcome problems associated with intramuscular quinine administration but the drug was sometimes expelled from the rectum; in addition the calculated intrarectal bioavailability was only about $40 \% .^{3}$

In contrast, multiple unit dosage forms (pellets or minitablets) offer a flexible dosing system. Since each individual unit contains a small amount of drug, the drug dose can be easily adjusted by measuring a specific volume (i.e. weight) of these multiparticulates depending on the patient's body weight. ${ }^{4}$

Quinine Sulphate is slightly soluble in water. An additional formidable challenge for an oral Quinine Sulphate formulation is its extremely bitter taste. Oral administration of bitter drugs with an acceptable degree of palatability is a key issue for the health care providers, especially for pediatric and geriatric patients. ${ }^{3,5}$

Concentration of as low as $0.025 \%(\mathrm{w} / \mathrm{v})$ solution was classified at the highest score on a bitter taste scale, only solutions below $0.001 \%$ were considered as having an acceptable bitter taste. Hence, taste masking of the oral formulations becomes imperative. Different techniques are available for taste masking but polymer coating is preferred as the Quinine Sulphate pellets being spherical in shape increase the efficiency of coating process. As the drug particles are individually coated the drug contact with taste bud is prevented, thus masking the bitter taste of drug. These SLNs can also be delivered in the form of nanosuspension or nanoemulsion for pediatric and geriatric patients. ${ }^{6,7}$

The drawbacks of conventional therapy for malaria viz. development of drug resistance, non specific targeting, subsequent toxicity due to high dose etc have shifted the focus on nanosized carriers which overcome the aforementioned problems. Considering the peculiar nature of malarial parasites, focus is shifted to developing lipid-based (e.g., liposomes, solid lipid nanoparticles and nano and microemulsions) and polymer based nanocarriers (nanocapsules and nanospheres). ${ }^{8}$

For oral treatment, the weight-adjusted doses of antimalarials in infants are similar to those used in adults.
However, the lack of infant formulations for the majority of antimalarials necessitates the division of adult formulations, which leads to inaccurate dosing. Furthermore, taste, volume and gastrointestinal tolerability are important determinants of treatment acceptability by children. Hence, there is an urgent need to develop oral formulations for antimalarials in order to improve the accuracy and reliability of dosing in children. ${ }^{3}$

In the present study, Quinine Sulphate loaded SLNs will be prepared by an ultrasonic-solvent emulsification technique and the physicochemical characteristics of the SLNs will be investigated. Formulation of Quinine Sulphate loaded SLNs will help to mask the bitter taste and provide dose precision. Solid lipid nanoparticles also provide a flexible system that allows dose adaptation according to the body weight. ${ }^{4}$

\section{MATERIAL AND METHODS}

\section{Materials}

Quinine Sulphate was purchased from S D Fine Chemicals Pvt. Ltd., Mumbai, India. Glyceryl monostearate and Pluronic F68 was obtained as a gift sample from Venus Ethoxyether, Goa, India. Pluronic F127 was obtained as a gift sample from Sigma Aldrich, USA. Tween 80 was purchased from Loba Chemicals Pvt. Ltd., Mumbai, India. Solvents were of analytical grade and produced commercially.

\section{Procedure for the preparation of Solid Lipid Nanoparticles}

SLNs were prepared by an ultrasonic-solvent emulsification technique. The desired amounts of Glyceryl monostearate (GMS) and Quinine Sulphate (QS) were mixed with trace amounts of methanol and choroform $(1: 1)$ and heated to $50^{\circ} \mathrm{C}$. Emulsifiers (Tween 80/ PF127/PF68) were dispersed in $10 \mathrm{~mL}$ distilled water with magnetic stirring at the same temperature. After evaporating most of the solvent, the water phase was added to the oil phase drop wise at $50^{\circ} \mathrm{C}$ followed by mechanical stirring for $10 \mathrm{~min}$, then the coarse emulsion was subjected to $600 \mathrm{~W}$ of ultrasonic treatment for 5 min using a high-intensity probe ultrasonicator (Q125, Rivotek, Kenya) with water bath $\left(0^{\circ} \mathrm{C}\right)$. The dispersions were immediately dispersed in bulks of distilled water $\left(0^{\circ} \mathrm{C}\right)$ followed by magnetic stirring to remove traces of organic solvents if any. After the dichloromethane had completely evaporated the QS-SLN suspensions were filtered through a $0.45 \mu \mathrm{m}$ membrane in order to remove the impurities. The QS-SLNs were stored at $4^{\circ} \mathrm{C} .{ }^{1,9}$ The composition of all the formulations is given in (Table 1).

\section{Particle size analysis and Polydispersity index}

The size distribution and polydispersity index (PDI) of the formulations was measured by Dynamic Light Scat- 
Table 1: Composition of Quinine Sulphate loaded solid lipid nanoparticle formulations

\begin{tabular}{|c|c|c|c|c|c|c|c|c|c|}
\hline \multirow{2}{*}{$\begin{array}{l}\text { Formulation } \\
\text { Ingredients }\end{array}$} & \multicolumn{9}{|c|}{ Formulation Code } \\
\hline & F1 & F2 & F3 & F4 & F5 & F6 & F7 & F8 & F9 \\
\hline $\begin{array}{l}\text { Quinine sulphate } \\
\text { (mg) }\end{array}$ & 300 & 300 & 300 & 300 & 300 & 300 & 300 & 300 & 300 \\
\hline $\begin{array}{l}\text { Glyceryl } \\
\text { monostearate (mg) }\end{array}$ & 900 & 900 & 900 & 900 & 900 & 900 & 900 & 900 & 900 \\
\hline Tween 80 (\%w/v) & 1.0 & 1.5 & 2.0 & - & - & - & - & - & - \\
\hline $\begin{array}{l}\text { Pluronic F127 } \\
(\% w / v)\end{array}$ & - & - & - & 1.0 & 1.5 & 2.0 & - & - & - \\
\hline Pluronic F68 (\%w/v) & - & - & - & - & - & - & 1.0 & 1.5 & 2.0 \\
\hline $\begin{array}{l}\text { Methanol \& } \\
\text { Chloroform (1:1) }\end{array}$ & q.s. & q.s. & q.s. & q.s. & q.s. & q.s. & q.s. & q.s. & q.s. \\
\hline
\end{tabular}

tering Particle Size Analyzer (Nano-flex, Microtrac Inc., USA). The range of the analyzer is $0.02 \mathrm{~nm}$ to $2.8 \mu \mathrm{m}$. Monodisperse samples have a lower PDI value, whereas higher value of PDI indicates a wider particle size distribution and the polydisperse nature of the sample. The usual range of PDI values: 0-0.05 (monodisperse standard), 0.05-0.08 (nearly-monodisperse), 0.08-0.7 (midrange polydispersity), $>0.7$ (very polydisperse)..$^{10,11}$

\section{Scanning Electron Microscopy (SEM)}

The shape of the SLNs was characterized by Scanning Electron Microscopy. The dried SLNs were mounted on metal stubs and coated with Platinum using a Sputter Coater JFC-1600 (JEOL, Japan) and were then observed under JSM-6360LV Scanning Electron Microscope JEOL, Japan.

\section{Entrapment efficiency and Drug Loading}

Formulation equivalent to $1 \mathrm{mg}$ was taken in an eppendorf tube and centrifuged (Superspin R-V/FA) at 15000 $\mathrm{rpm}$ for $1 \mathrm{hr}$ and the pellet obtained was dissolved in 1 $\mathrm{ml}$ ether to dissolve the lipid and $1 \mathrm{ml}$ of hot water $\left(100^{\circ} \mathrm{C}\right)$ was added to dissolve the drug and precipitate the lipid. It was again centrifuged at $15000 \mathrm{rpm}$ for 1 hr. Concentration of Quinine Sulphate in the supernatant was determined at $330 \mathrm{~nm}$ using UV-visible spectrophotometer (Shimadzu UV-1700 Pharmaspec, S.No. A11024504164, Japan). The percentage entrapment efficiency and percentage drug loading were determined by taking the appropriate formulae. ${ }^{12,13}$

\section{Percentage Yield}

Fixed volumes of SLN dispersions were centrifuged at $9000 \mathrm{RPM}$ for $30 \mathrm{~min}$ at $15^{\circ} \mathrm{C}$. The obtained sediment was dried and weighed and the percentage yield was calculated.

\section{DSC Analysis}

Differential Scanning Calorimetry (DSC) analysis was performed on a Differential Scanning Calorimeter
(Model DSC-60) Shimadzu Co., Japan). Approximately $5 \mathrm{mg}$ of the formulation F9 was weighed into an aluminium pan and sealed hermetically. DSC scan was recorded from 30 to $300^{\circ} \mathrm{C}$ at a heating rate of $10^{\circ} \mathrm{C} /$ min under a nitrogen purge, using an empty pan as reference. $^{14,15}$

\section{Zeta potential}

Zeta potential is the difference in the potential between the surface of tightly bound layer (shear plane) and the electroneutral region of the solution. It is important parameter to analyze long term stability of nanoparticles. A solid particle (colloid) dispersed in a liquid media can acquire a surface charge by the adsorption of ions present in the solution, by the ionization of functional groups on the surface of the particle or due to the difference in dielectric constant between the particle and the medium. ${ }^{16}$ Zeta potential was measured by using MICROTRAC Zetatrac NPA152-31A.

\section{Evaluation of taste masking efficiency}

The taste masking efficiency of SLNs was determined using Franz Diffusion cell. The receptor cell consisted of Phosphate Buffer Saline ( $\mathrm{pH}$ 6.8). An accurately weighed amount of QS-SLN dispersion equivalent to $10 \mathrm{mg}$ of drug was transferred to the dialysis membrane (Hi-media, Mumbai, India) with molecular weight cutoff 12,000-14,000 Dalton placed between the donor and the receptor compartment. $1 \mathrm{ml}$ samples were withdrawn from the receptor compartment at predetermined time intervals and analyzed spectrophotometrically at $330 \mathrm{~nm}$. The medium was replaced after each sampling with PBS $\mathrm{pH}$ 6.8. ${ }^{17,18}$

\section{In vitro drug release}

The In vitro release of QS from different SLN dispersions was determined using the dialysis bag diffusion technique. An accurately weighed amount of QS-SLN dispersion equivalent to $10 \mathrm{mg}$ of drug was transferred to a dialysis bag (Hi-media, Mumbai, India) with molecu- 
Table 2: Particle Size, Polydispersity Index, Zeta Potential, Entrapment Efficiency, Drug Loading and Percentage Yield of Formulations

\begin{tabular}{|c|c|c|c|c|c|c|}
\hline Formulation & $\begin{array}{c}\text { Mean Particle } \\
\text { Size }(\mathrm{nm})^{*}\end{array}$ & $\begin{array}{l}\text { Polydispersity } \\
\text { index (PI) * }\end{array}$ & $\begin{array}{c}\text { Zeta Potential } \\
(\mathrm{mV})^{*}\end{array}$ & $\begin{array}{c}\text { Entrapment } \\
\text { efficiency }(\%) \#\end{array}$ & $\begin{array}{c}\text { Drug loading } \\
(\%)^{\#}\end{array}$ & Yield (\%) \# \\
\hline F1 & $214.3 \pm 5.90$ & $0.942 \pm 0.024$ & $-29.09 \pm 0.80$ & 87.07 & 29.02 & 68.33 \\
\hline F2 & $186.9 \pm 8.51$ & $0.518 \pm 0.003$ & $-30.31 \pm 1.24$ & 83.84 & 27.95 & 66.16 \\
\hline F3 & $116.4 \pm 14.19$ & $0.516 \pm 0.014$ & $-32.16 \pm 1.31$ & 81.97 & 27.32 & 62.16 \\
\hline F4 & $66.2 \pm 10.92$ & $0.370 \pm 0.010$ & $-31.52 \pm 1.52$ & 88.95 & 29.65 & 69.91 \\
\hline F5 & $265.7 \pm 13.12$ & $0.867 \pm 0.001$ & $-32.63 \pm 1.66$ & 91.67 & 30.56 & 73.58 \\
\hline F6 & $692.0 \pm 9.96$ & $1.114 \pm 0.017$ & $-34.56 \pm 0.10$ & 92.35 & 30.79 & 76.25 \\
\hline F7 & $182.2 \pm 8.72$ & $0.496 \pm 0.004$ & $-33.13 \pm 0.82$ & 89.80 & 29.94 & 72.50 \\
\hline F8 & $159.0 \pm 7.11$ & $0.638 \pm 0.018$ & $-34.16 \pm 0.83$ & 93.03 & 31.01 & 74.33 \\
\hline F9 & $145.8 \pm 6.49$ & $0.500 \pm 0.001$ & $-36.88 \pm 1.12$ & 94.56 & 31.52 & 77.58 \\
\hline
\end{tabular}

lar weight cutoff 12,000-14,000 Dalton and sealed. The sealed bag was then suspended in USP Type II Apparatus containing $900 \mathrm{ml}$ of $0.1 \mathrm{~N} \mathrm{HCl}$ and stirred at a constant speed of $50 \mathrm{rpm}$ at $37^{\circ} \mathrm{C} \pm 0.5^{\circ} \mathrm{C}$. Aliquots were withdrawn at predetermined intervals from the receptor compartment and replaced with fresh buffer. The drug release was determined spectrophotometrically by measuring the absorbance at $330 \mathrm{~nm}$ using the respective receptor medium as a blank, to calculate the amount of drug released from the nanoparticles. ${ }^{17}$

\section{RESULTS AND DISCUSSION}

\section{Evaluation of Formulated SLNS}

\section{Particle size, Size distribution and Polydispersity index}

Particle size measurement was required to confirm the production of the particles in nano-range. Particle size data for the SLNs of quinine sulphate is shown in (Table 2). The mean particle size for formulations F1 to F9 varied in range of 66.2 to $673.8 \mathrm{~nm}$. The particle size analysis reveals that the size reduction was due to speed. As the lipid concentration was increased, more particles were aggregated resulting in an increased particle size. In general, mean hydrodynamic diameter of the particle decreased with an increase in the surfactant concentration. This observation was in conformity with the findings of Ekambaram et al. SLN dispersion prepared using poloxamer 188 as a stabilizer showed lower particle size than the other surfactants. This result could be explained due to the higher HLB value of poloxamer 188 when compared to tween 80 and poloxamer $407 .{ }^{9}$

Polydispersity index (PDI) indicates the width of the particle size distribution, which ranges from 0 to 1 . A monodisperse sample indicates PDI value nearer to
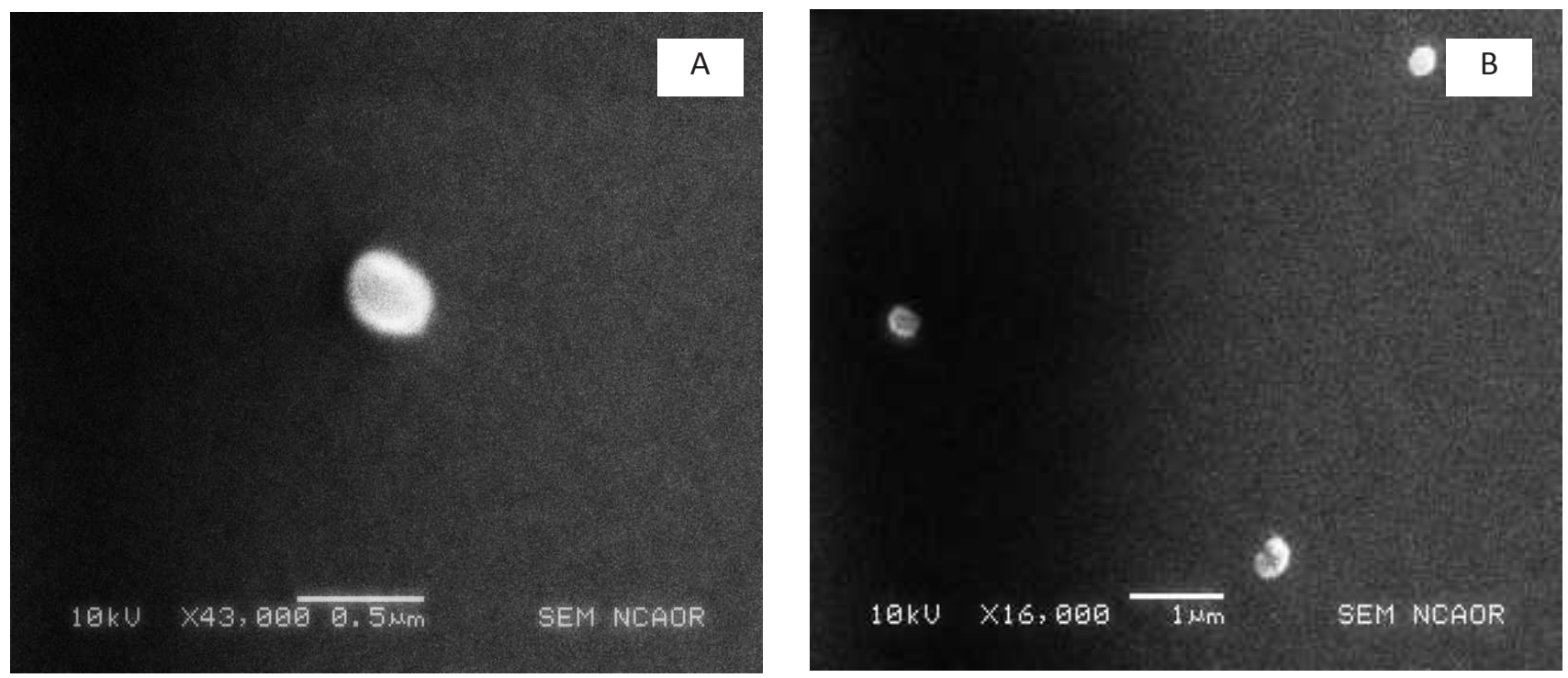

Figure 1: (a) and (b) SEM images of optimized formulation F9 containing 2\% Poloxamer 188 at different resolutions 
0 . However, PDI $<1$ indicates polydisperse samples. Therefore, PDI measurement was essential to confirm the size distribution of the particles. The mean polydispersity index values for the drug loaded SLN formulations varied in the range of $0.370-1.114$ as tabulated in (Table 2). ${ }^{19}$

\section{Zeta $(\zeta)$ potential}

Zeta potential $( \pm)$ indicates the degree of repulsion between close and similarly charged particles in the dispersion. This repulsion force prevents aggregation of the particles. Therefore, it is a useful parameter to predict the physical stability of the SLN dispersions. Values more than $+20 \mathrm{mV}$ or less than $-20 \mathrm{mV}$ predict good physical stability of nanoparticle dispersion. The $\zeta$ Potential values of the formulations were found to be between -29.09 to $-36.88 \mathrm{mV}$ (Table 2). It predicts good particle stability because the repulsive forces prevent aggregation with aging. ${ }^{19}$

\section{Scanning Electron Microscopy}

SEM images of all the formulations suggest that the formulations were in nano range. The particles were oval in shape and discrete. SEM images of the optimized formulation F9 containing 2\% PF 68 are shown in (Figure 1a and 1b). It can be seen that the surfactant helps to form oval particles with a smooth surface and prevents agglomeration of the particles.

\section{Entrapment efficiency and Drug Loading}

The experimental results indicate that the concentration of surfactant has critical effect on the quinine sulphate

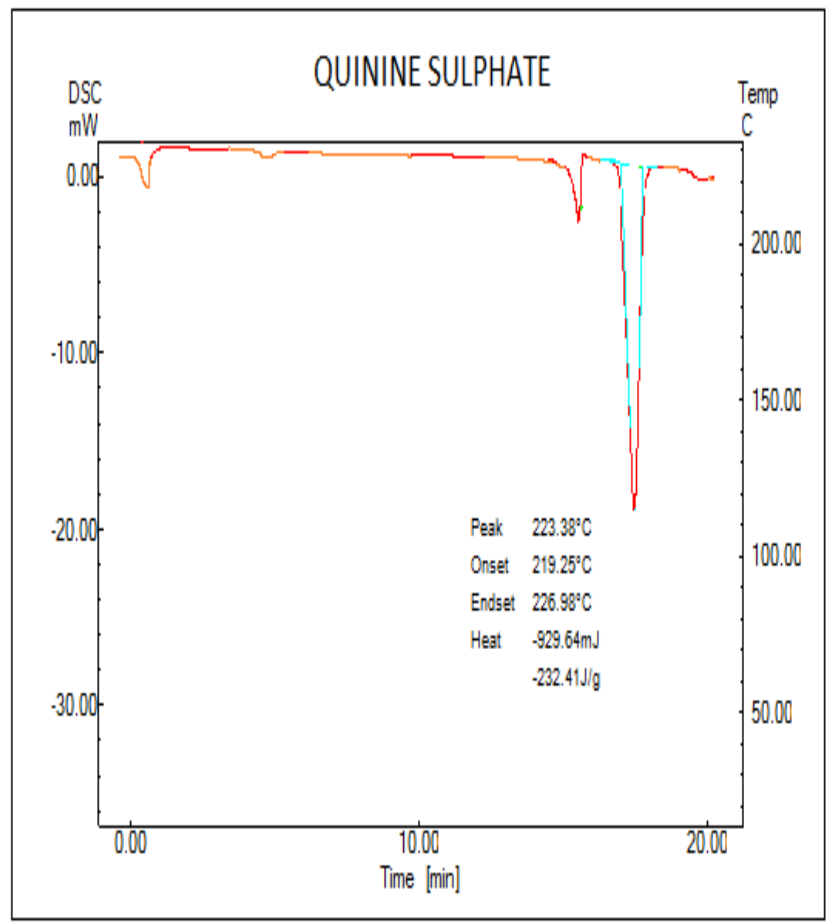

incorporation efficacy. The values of drug entrapment efficiency are shown in (Table 2). The entrapment efficiency was found to be in the range of $81.97 \%$ to $94.55 \%$. A higher concentration of surfactant (poloxamers) helps lipid to encapsulate quinine sulphate effectively. However, it can be seen that increase in the concentration of Tween 80 causes decrease in the entrapment efficiency. This can be attributed to the solubilization effect of Tween 80 on the solid lipid nanoparticles.

The experimental results indicate that the concentration of surfactant has critical effect on the drug loading. The values of drug loading are shown in (Table 2). The drug loading was found be in the range of $27.32 \%$ to $31.51 \%$.

\section{Percentage Yield}

The Percentage Yield of the SLNs increased with increase in the surfactant concentration in case of poloxamers 188 and 407, but decreased in case of Tween 80 (Table 2). This can be attributed to the solubilization effect of Tween 80 which leads to solubilization of the SLNs.

\section{Differential Scanning Calorimetry}

The DSC analysis was performed on the optimized formulation i.e. F9. The optimized formulation was selected based upon the other evaluation tests. The DSC thermograms of pure drug and the optimized SLN formulation are depicted in (Figure $2 \mathrm{a}$ and $2 \mathrm{~b}$ ) respectively. The DSC thermogram of quinine sulphate exhibited a single sharp exothermic peak at $223.38^{\circ} \mathrm{C}$ corresponding to its melting transition temperature. This peak was

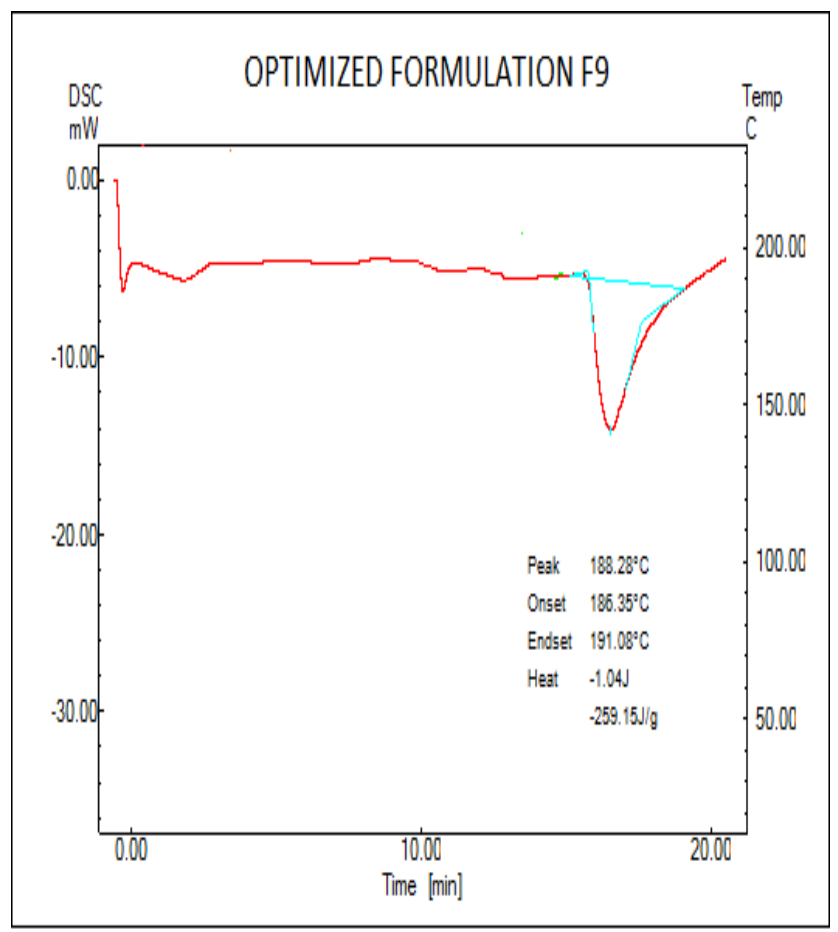

Figure 2: (a) DSC thermogram of pure drug (Quinine sulphate) and (b) DSC thermogram of the optimized formulation F9 containing $2 \%$ Poloxamer 188 
Table 3: In vitro release data of formulations F1-F9 at pH 6.8

\begin{tabular}{|l|c|c|c|c|c|c|c|c|c|c|}
\hline \multicolumn{7}{|c|}{ Table 3: In vitro release data of formulations F1-F9 at pH $\mathbf{6 . 8}$} \\
\hline Time $(\mathbf{m i n})$ & $\begin{array}{c}\text { Pure } \\
\text { Drug }\end{array}$ & F1 & F2 & F3 & F4 & F5 & F6 & F7 & F8 & F9 \\
\hline $\mathbf{0}$ & 0 & 0 & 0 & 0 & 0 & 0 & 0 & 0 & 0 & 0 \\
\hline $\mathbf{5}$ & 8.12 & 0 & 0 & 0 & 0 & 0 & 0 & 0 & 0 & 0 \\
\hline $\mathbf{1 0}$ & 11.63 & 0 & 0 & 0 & 0 & 0 & 0 & 0 & 0 & 0 \\
\hline $\mathbf{1 5}$ & 14.38 & 0.03 & 0 & 0 & 0 & 0.02 & 0 & 0 & 0 & 0 \\
\hline $\mathbf{2 0}$ & 15.50 & 0.04 & 0.02 & 0 & 0.03 & 0.05 & 0 & 0.02 & 0 & 0 \\
\hline $\mathbf{2 5}$ & 18.48 & 0.06 & 0.02 & 0.03 & 0.05 & 0.06 & 0.03 & 0.04 & 0.02 & 0 \\
\hline $\mathbf{3 0}$ & 21.46 & 0.1 & 0.05 & 0.05 & 0.09 & 0.08 & 0.07 & 0.09 & 0.04 & 0.02 \\
\hline "Data are expressed as Mean (n=3) & & & & & & & \\
\hline
\end{tabular}

not observed in the thermogram of the optimized drug loaded SLNs. No melting point typical of Quinine Sulphate was detected in the DSC curve of the drug loaded SLNs, thus no free drug crystals were reported in the system. The broadened and shifted peaks suggests that the absence of the crystalline form of the drug in the drug-loaded SLNs, indicating that the drug was present as a molecular dispersion in the lipid matrix. Incorporation of the drug inside the lipid matrix results in an increase in the number of defects in the lipid crystal lattice, and hence causes a decrease in the melting point in the final SLN formulation.

\section{In vitro evaluation of taste masking efficiency}

In vitro evaluation of taste masking efficiency was carried out by determining the amount of drug released from the SLNs of Quinine Sulphate in simulated salivary fluid ( $\mathrm{pH}$ 6.8). It was performed using Franz Diffusion Cell. The results are shown in (Table 3). The formulations showed negligible drug release at the end of 30 min when compared with pure drug. The results are in conformity with the findings of Patravale $e t$ al. wherein no drug release was observed in simulated salivary fluid pH 6.8 indicating that significant amount of drug does not come in contact with the saliva to generate a bitter taste. Formulation F9 showed the lowest release at the end of 30 min indicating better taste masking efficiency of the formulation. ${ }^{17}$

\section{In vitro drug release}

In vitro drug release from the SLNs of quinine sulphate in $0.1 \mathrm{~N} \mathrm{HCl}$ buffer ( $\mathrm{pH} 1.2$ ) was performed using dialysis bag technique. The In vitro drug release profile of SLNs of quinine sulphate shown in (Table 4). Surfactant concentration has a direct effect on the drug release profile. It shows that almost $100 \%$ of the drug is released at the end of $90 \mathrm{~min}$ at $\mathrm{pH} 1.2$ indicating that the formulation of QS-SLNs does not hamper the release of quinine sulphate in acidic medium. The results are in conformity with the findings of Patravale et al. where they observed complete elution of the drug at $\mathrm{pH}$ 1.2. The formulations showed an increase in the percentage drug release with increase in the surfactant concentration. This can be attributed to the solubilization effect of the surfactants. The increase in the surfactant concentration helps the drug to go into solution. ${ }^{17}$

\section{Selection of the optimized formulation}

Formulation F9 having $2 \%$ w/v PF 68 was found to be the optimized formulation based on its particle shape and size, polydispersity index, zeta potential, entrap-

\begin{tabular}{|l|c|c|c|c|c|c|c|c|c|c|}
\hline \multicolumn{7}{|c|}{ Table 4: In vitro release data of formulations F1-F9 at pH 1.2} \\
\hline Time (min) & $\begin{array}{c}\text { Pure } \\
\text { Drug }\end{array}$ & F1 & F2 & F3 & F4 & F5 & F6 & F7 & F8 & F9 \\
\hline $\mathbf{0}$ & 0 & 0 & 0 & 0 & 0 & 0 & 0 & 0 & 0 & 0 \\
\hline $\mathbf{5}$ & 31.84 & 31.07 & 29.39 & 27.7 & 32.45 & 32.91 & 27.4 & 30.46 & 27.40 & 30.31 \\
\hline $\mathbf{1 0}$ & 41.07 & 41.06 & 37.98 & 35.82 & 39.54 & 44.14 & 35.66 & 38.76 & 35.05 & 39.06 \\
\hline $\mathbf{1 5}$ & 50.40 & 52.69 & 49.57 & 51.06 & 52.68 & 54.58 & 47.69 & 46.38 & 45.84 & 52.5 \\
\hline $\mathbf{3 0}$ & 71.46 & 70.4 & 67.72 & 65.85 & 65.81 & 71.7 & 64.13 & 58.06 & 56.14 & 68.84 \\
\hline $\mathbf{4 5}$ & 80.35 & 80.05 & 78.1 & 76.83 & 75.86 & 77.69 & 75.69 & 72.16 & 68.69 & 80.61 \\
\hline $\mathbf{6 0}$ & 89.79 & 88.41 & 84.61 & 85.62 & 83.72 & 84.04 & 86.31 & 83.65 & 88.87 & 89.6 \\
\hline $\mathbf{7 5}$ & 95.19 & 94.41 & 92.86 & 91.59 & 87.53 & 92.28 & 93.36 & 93.43 & 91.06 & 95 \\
\hline $\mathbf{9 0}$ & 100.16 & 97.26 & 99.21 & 99.61 & 96.73 & 98.47 & 98.49 & 96.57 & 98.46 & 99.99 \\
\hline *Data are expressed as Mean (n=3) & & & & & & & \\
\hline
\end{tabular}


ment efficiency, drug loading, taste masking efficiency and percentage drug release (\%CDR) when compared with other formulations.

\section{Dose in pediatric patients}

The adult dose of Quinine Sulphate is $300 \mathrm{mg}$. However, the pediatric dose is $10 \mathrm{mg} / \mathrm{kg}$. $10 \mathrm{ml}$ of the prepared SLN dispersion contains around $300 \mathrm{mg}$ of quinine sulphate. Therefore, $1 \mathrm{ml}$ shall contain $30 \mathrm{mg}$ of the drug. In case of a pediatric patient weighing $3 \mathrm{~kg}$, the dose would be $30 \mathrm{mg}$ i.e. $1 \mathrm{ml}$ of the SLN dispersion.

\section{CONCLUSION}

Taste masked solid lipid nanoparticles of Quinine Sulphate can be successfully formulated by ultrasonic solvent emulsification technique using selected lipid and surfactants for better patient compliance and to provide dose precision and a flexible system that allows dose adaptation according to the body weight. The mean hydrodynamic diameter of the particle decreased whereas the entrapment efficiency increased with an increase in the surfactant concentration. The zeta potential values predicted good particle stability because the repulsive forces prevent aggregation with aging. The DSC thermograms suggest that the drug was molecularly dispersed in the lipid matrix. Quinine Sulphate release kinetics was dependent upon the surfactant type and concentration. Higher surfactant concentration showed faster In vitro release. The formulations showed negligible release at $\mathrm{pH} 6.8$ and almost $100 \%$ release at $\mathrm{pH}$ 1.2 , which is desirable so as to mask the taste by delaying the release during administration without hampering the drug release in stomach. Thus, it can be concluded that Quinine Sulphate was proven to be a suitable candidate for formulating solid lipid nanoparticles to achieve better patient compliance among paediatrics and geriatric population by masking the bitter taste and avoiding the difficulty in swallowing. The solid lipid nanoparticles would also offer more flexibility to body weight dose adaptation in pediatrics. Future work can be taken up with respect to in vivo taste evaluation, In vivo pharmacokinetics and pharmacodynamics and long term stability studies can be carried out in order to characterize the delivery system for clinical use.

\section{ACKNOWLEDGEMENT}

The authors would sincerely like to thank S D Fine Chemicals, Mumbai for providing quinine sulphate and Venus Ethoxyether, Goa for providing gift samples of glyceryl monostearate and pluronic F68. We would also like to thank Dr. Rahul Mohan and his team at National
Centre for Antarctic and Ocean Research, Goa for providing SEM facilities. The authors are also grateful to KLE University's Dr. Prabhakar Kore Basic Science Research Centre, Belgaum for providing the laboratory facilities.

\section{CONFLICTS OF INTEREST}

The authors declare that they have no competing interests.

\section{REFERENCES}

1. Luo YF, Chen DW, Ren LX, Zhao XL, Qin J. Solid Lipid Nanoparticles for Enhancing Vinpocetine's Oral Bioavailability. J Control Release. 2006; 114(1): 53-9.

2. Sweetman SC. Martindale: The Complete Drug Reference. $37^{\text {th }}$ ed. London: Pharmaceutical Press. 2011.

3. Kayumba PC. Taste - masked Quinine Formulations for Flexible Pediatric Drug Dosing in Oral Treatment of Malaria [PhD thesis]. Ghent, Rwanda: National University of Rwanda; 2007.

4. Momin M, Rathod S, Kar S. Taste Masking Techniques for bitter Drugs- an Overview. Int J Pharm Tech. 2012; 4(2): 2100-18.

5. Indian Pharmacopoeia. Ghaziabad. Indian Pharmacopoeia Commission. 2007; 3: 1025-6

6. Kashanian S, Azandaryani AH, Derakhshandeh K. New Surface-Modified Solid Lipid Nanoparticles using N-Glutaryl phosphatidylethanolamine as the Outer Shell. Int J Nanomed. 2011; 6: 2393.

7. Thatipamula RP, Palem CR, Gannu R, Mudragada S, Yamsani MR. Formulation and in vitro characterization of Domperidone loaded SLN and NLC. DARU. 2011; 19(1): 23.

8. Santos-Magalhães NS, Furtado Mosqueira VC. Nanotechnology applied to the treatment of malaria. Adv Drug Del Rev. 2010; 62(4): 560-75.

9. Ekambaram P, Abdul HSA. Formulation and evaluation of SLNs of Ramipril. J Young Pharm. 2011; 3(3): 216-20.

10. Vijayan V, Rao SD, Jayachandran E, Anburaj J. Preparation and characterization of Anti Diabetic Drug Loaded Solid lipid Nanoparticles. J Inno Trends Pharm Sci. 2010; 1(8): 320-8.

11. Wolfgang S. Sample preparation in Light Scattering from Polymer Solutions and Nanoparticle Dispersions. Springer Berlin Heidelberg $\mathrm{GmbH} \& \mathrm{Co}$; $\mathrm{K}$ 2007. p. 43-4.

12. Nair R, Vishnupriya K, Kumar AKS, Badivaddin T, Sevukarajan M. Formulation and Evaluation of Solid Lipid Nanoparticles of Water Soluble Drug: Isoniazid. J Pharm Sci Res. 2011; 3(5): 1256-64.

13. Sharma R, Yasir M, Bhaskar S, Asif M. Formulation and evaluation of Paclitaxel loaded PSA-PEG nanoparticles. J Applied Pharm Sci. 2011; 1(5): 96-8.

14. Yang C, Zhao X, Hu H, Li K, Sun X, Li L, Chen D. Preparation, Optimization and Characteristic of Huperzine A Loaded Nanostructured Lipid Carriers. Chem Pharm Bull. 2010; 58(5): 656-61.

15. Edavalath S, Prakasham K, Rao B, Divakar G. Formuation development and Optimization of controlled porosity osmotic pump tablets of Diclofenac sodium. Int J Pharm Sci. 2011; 3(1): 80-7.

16. Martin A. Physical Pharmacy. 4th ed. Lippincott Williams \&amp; Wilkins, Philadelphia, PA; 1993. p. 386-8.

17. Patravale VB, Prabhu NB. Taste Masking of Quinine Sulphate. Indian J Pharm Sci. 2005; 67(2): 233-5.

18. Jadon RS, Nayak S, Amlan S, Vaidya VD, Khemariya P, Sumbhate S. Taste masking of Lornoxicam by polymer carrier system and formulation of oral disintegrating tablets. Int J Drug Delivery. 2011; 1(1): 127-31.

19. Das S, Ng W, Tan RBH. Are Nanostructured lipid carriers (NLCs) better than solid lipid nanoparticles (SLNs): Development, characterizations and comparative evaluations of clotrimazole-loaded SLNs and NLCs. Eur J Pharm Sci. 2012; 47(1): 139-51. 